\title{
Graphene oxide-tripolyphosphate hybrid used as a potent sorbent for cationic dyes
}

\author{
Paul N. Diagboya ${ }^{a, b}$, Bamidele I. Olu-Owolabi ${ }^{b}$, Ding Zhou ${ }^{a}$, Bao-Hang Han ${ }^{a, *}$ \\ ${ }^{a}$ National Center for Nanoscience and Technology, Beijing 100190, China \\ b Department of Chemistry, University of Ibadan, Ibadan, Nigeria
}

A R T I C L E I N F O

Article history:

Received 19 February 2014

Accepted 22 July 2014

Available online 30 July 2014

\begin{abstract}
A B S T R A C T
Graphene oxide-tripolyphosphate material (GPM) was synthesized through an ethanolamine (EA) mediated graphene oxide (GO) self-assembly. The synthesis route to GPM is simple and benign. GPM was composed of GO nanosheets as building blocks and the tripolyphosphate as cross-linkers and chelators of cations in solutions. GPM showed higher potency for adsorption of cationic dyes than anionic dyes, and the adsorption process was through electrostatic and $\pi-\pi$ interactions. Adsorption was spontaneous and exothermic, and the adsorption capacity of GPM for cationic dyes (>2540 $\left.\mathrm{mg} \mathrm{g}^{-1}\right)$ far exceeded those reported in literature for GO materials.
\end{abstract}

(c) 2014 Elsevier Ltd. All rights reserved.

\section{Introduction}

The magnitude of surface water pollution, especially from industries, is enormous and remediation is seemingly intractable because of the diverse sources of pollution. The current technologies used in industrial wastewater treatment systems are not effective enough. Hence, technologies that can achieve high efficiency in wastewater treatment should be considered with priority since treatment of industrial effluents before disposal into surface water bodies would be an effective method to reduce surface water pollution [1].

Advances in graphene chemistry have triggered enormous interests in the applied science fields [2]. The concept of the synergistic combination of different molecules in one single product has become a very successful approach in materials science for achieving highly efficient and specific goals in materials' property. This includes the making of materials with new structural and functional properties superior to those of the individual pure components. Such focus on the physical properties of graphene has resulted in the discovery of plethora of new materials leading to the fabrication of prototype devices such as field-effect transistors [3] and extraordinary high sensitivity chemical sensors [4]. However, despite the intriguing properties anticipated for functionalized graphene, chemical modification of graphene for environmental application remains largely underexplored. In this study, we reported the functionalization of graphene oxide (GO) with tripolyphosphate (TPP) which has been used for dye removal from water. TPP has been recognized as a very potent chelating agent for cations in solution. Here it was coupled to GO through the initiator ethanolamine (EA), in a reaction similar to the $\mathrm{GO}$ amidation reaction of Yang et al. [5]. The amine group of EA was attached to the GO sheet through the carboxylic acid functional group following the $\mathrm{N}$-(3-dimethylaminopropyl)- $\mathrm{N}^{\prime}$-ethylcarbodiimide hydrochloride (EDC) chemistry: a similar reaction to that described by Liu et al. [6]. The EDC was the coupling agent between the amine of EA and the carboxylic group of GO. The coupled amine group can become easily protonated, thus acquiring a positive charge. The TPP assembly was formed by electrostatic interaction between the negatively charged TPP terminals and the positively protonated amine groups on GO

\footnotetext{
* Corresponding author.

E-mail address: hanbh@nanoctr.cn (B.-H. Han). http://dx.doi.org/10.1016/j.carbon.2014.07.057 0008-6223/@ 2014 Elsevier Ltd. All rights reserved.
} 
sheet. Several GO sheets are cross-linked in this manner to form the GO-TPP hybrid material (GPM). A schematic representation of the synthesis is shown in Fig. 1. This new graphene material (GPM) is amphiphilic containing uncharged and aromatic graphene portions as well as the charged groups such as the hydroxyl, phosphate, and carboxylic groups. Due to the small size of the tripolyphosphate molecule the assembly is believed to be somewhat packed together in a sponge-like manner.

GPM adsorbent assembly was formed spontaneously at the basal plane or edges of graphene or GO due to chemical or physical interactions of molecules with the graphene or GO sheets, without high-vacuum or high-temperature processing [2]. These interactions occur mainly on the oxygen species through such groups as hydroxyl $(-\mathrm{OH})$ or carboxyl $(-\mathrm{COOH})$ which are necessary for the molecules to covalently bond to the surface [7].

The synthesized GPM was used for the removal of two different categories of dyes from water: cationic (methyl blue $\mathrm{MB}$, methyl violet - MV, and basic fuchsine - BF) and anionic (methyl orange - MO, alizarin yellow - AY, amaranth - AM) dyes. Cationic and anionic dyes are commonly used in the textile industries; though currently the use of a single cationic or anionic dye is limited, and the commonly used reactive dyes are composed of both cationic and anionic functional moieties. These functional moieties make the reactive dyes react covalently with the fabric as well as causing better exhaustion, fixation, and fastness of these dyes. A major challenge of industries involved with reactive dyes is effluent disposal. Most effluents are discharged into water bodies, and the major after-use challenges of these dyes are environmental. They are known carcinogens in biota, for example BF is a known category 2 carcinogen, irritating to respiratory system and eyes, and forms methemoglobin (an altered form of hemoglobin that cannot bind oxygen) with the human hemoglobin, thus leading to suffocation.

The aim of this study was to synthesize and use GPM adsorbent for cleanup of simulated cationic and anionic dyes polluted water. Since reactive dyes and their intermediates contain both moieties, data from the study was used to understand which functional moieties were involved in the adsorption process as well as explain the dynamics of the adsorption process.

\section{Experimental}

\subsection{Preparation of GO and GPM}

The chemical exfoliation of natural flake graphite was carried out by modified Hummers' method in which a very long oxi-
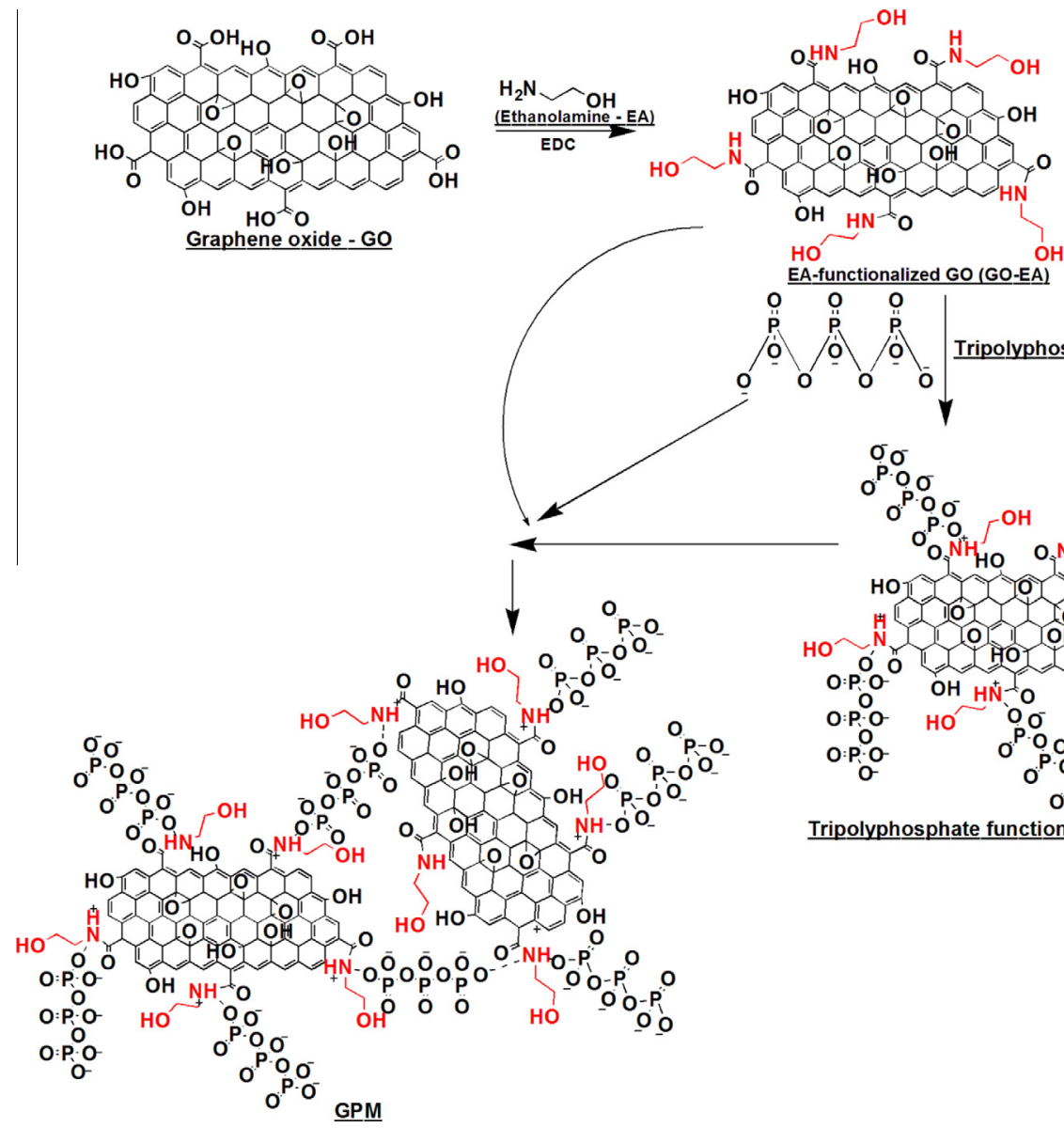

GPM

Fig. 1 - Schematics for the modification of GO with TPP using the EA initiator. (A color version of this figure can be viewed online.) 
dation period was combined with a multi-cycle purification process [8-10]. For the GPM synthesis, aqueous exfoliated GO suspension $\left(10.0 \mathrm{~g}, 13.26 \mathrm{mg} \mathrm{mL}^{-1}\right)$ and ultra-pure water (40 mL) were loaded into a $300 \mathrm{~mL}$ conical flask. This was sonicated at $40^{\circ} \mathrm{C}$ for $0.5 \mathrm{~h}$. Then $100 \mathrm{mg}$ of EDC and $2 \mathrm{~mL}$ EA were added into the conical flask and stirred for $0.5 \mathrm{~h}$. Sodium NaTPP solution $(0.25 \mathrm{M}, 20 \mathrm{~mL})$ was added and stirring continued for $2 \mathrm{~h}$. After stirring, the mixture was then kept quiescent for $5 \mathrm{~h}$, and the graphene tripolyphosphate assembly was collected by centrifugation, washed thrice with ultra-pure water, filtered and vacuum dried (Materials and methods in the Supplementary Materials).

\subsection{Instrumental characterization}

GPM was characterized by Spectrum One Fourier transform infrared (FTIR) spectrometer (PerkinElmer Instruments Co. Ltd., USA); Renishaw inVia Raman spectrometer (Renishaw plc, UK); scanning electron microscopy (SEM) images were observed on a Hitachi S-4800 scanning electron microscope at an accelerating voltage of $6 \mathrm{kV}$ (Hitachi Ltd., Japan); Philips X'Pert PRO X-ray diffraction instrument (PANalytical B.V., Netherlands); and Micromeritics ASAP $2020 \mathrm{M}+\mathrm{C}$ accelerated surface area and porosimetry analyzer (Micromeritics Instrument Corporation, USA). GPM was also subjected to thermo-gravimetric analysis (TGA) using Pyris Diamond Thermogravimetric/differential thermal analyzer (PerkinElmer Instruments Co. Ltd., USA), while the dye adsorptions were monitored using quartz cells with $1 \mathrm{~cm}$ path length on a PerkinElmer Lambda 950 UV-vis-NIR spectrophotometer (PerkinElmer Instruments Co. Ltd., USA) (details in the Supplementary Materials).

\subsection{Dye adsorptions}

Batch equilibrium adsorption experiments were done to determine the dye adsorption characteristics of GPM. The effects of time, $\mathrm{pH}$, dye concentration, and temperature on dye adsorption were ascertained using dye concentration of $2000 \mathrm{mg} \mathrm{L}^{-1}$ and $5.0 \mathrm{mg}$ of GPM. Where necessary, working solutions of lower concentrations were prepared from this concentration and used as soon as possible. The adsorption time ranged from 0 to $180 \mathrm{~min}$; $\mathrm{pH}$ from 3 to 11 ; and temperatures used were 15,25 , and $40^{\circ} \mathrm{C}$. The dye-GPM mixtures were shaken at the desired temperature in a temperature controlled shaker during the course of the sorption experiments. The tubes were withdrawn from the shaker at the appropriate time interval, centrifuged at $9000 \mathrm{rpm}$ for $10 \mathrm{~min}$ and the concentrations of dye in the clear upper solutions were determined at 600, 465, 374, 521, 584, and $544 \mathrm{~nm}$ for methyl blue, methyl orange, alizarin yellow, amaranth, methyl violet, and basic fuchsine dyes, respectively.

The extents of dye adsorptions on GPM were calculated using Eq. (1).

$q_{\mathrm{e}}=\frac{\left(C_{\mathrm{o}}-\mathrm{C}_{\mathrm{e}}\right) \mathrm{V}}{m}$

where $q_{\mathrm{e}}, C_{\mathrm{o}}$ and $C_{\mathrm{e}}$ are the amount of dye adsorbed $\left(\mathrm{mg} \mathrm{g}^{-1}\right)$, the initial and final dye concentrations in the solution $\left(\mathrm{mg} \mathrm{L}^{-1}\right)$, respectively; and $\mathrm{V}(\mathrm{mL})$ and $m(\mathrm{mg})$ are the volume of dye solution used and mass of GPM used for each adsorption, respectively.The Lagergren pseudo-first-order (PFO) and pseudo second-order (PSO) rate models as well as the Langmuir and Freundlich adsorption isotherms were used to describe the adsorption data. The BF dye thermodynamic adsorption parameters - enthalpy $\left(\Delta H^{\circ}\right)$, entropy $\left(\Delta S^{\circ}\right)$, Gibbs free energy $\left(\Delta G^{\circ}\right)$ and equilibrium constant $\left(K_{c}\right)$, were also evaluated from the adsorption data at 288, 298, and $313 \mathrm{~K}$ (Supplementary Materials).

\section{Results and discussion}

GO was employed in this modification instead of graphene because of the array of possible chemical reactions that are associated with the different oxygenated species on its surface [11], thus making GO significantly more versatile than graphene in this regard. This is an important advantage that allows the exploration of new routes for the immobilization of initiator molecules such as EA. Such initiator molecules are necessary due to the fact that despite the huge dimensions of the GO, in principle, no steric hindrance is expected as the polymer chains grow from the GO surface [12]. The GPM synthesis can be explained as follows: GO possesses considerable amount of oxygen containing groups, such as carboxyl, hydroxyl, and epoxide, on its surfaces, impacting the ability of even dispersion of GO in polar solutions because of these associated charged groups. Reaction of the GO terminal carboxylic group with EA enhances the dispersion of GO in water due to the large difference in size of the GO and EA. However, addition of TPP initiates the hybrid of these evenly dispersed GO sheets in solution due to the electrostatic interaction of the positively charged $-\mathrm{NH}_{3}^{+}$of EA functionalized GO and the negatively charged phosphate group of TPP. A synthesis pathway for this reaction showing GPM selfassembly obtained by reacting TPP with GO through the initiator molecule, EA, was proposed (Fig. 1). The positively charged amine group in the assembly provides spontaneous strong chemisorption point for TPP [13] resulting from charge transfer between the $-\mathrm{PO}_{3}^{-}$and $-\mathrm{NH}_{3}^{+}$. In this way, there are intra- and inter-molecular linkages created between both negatively charged ends of TPP and the positively charged amine groups on two GO nanosheets.

Fourier transform infrared (FTIR) spectra (Fig. 2a) showed the functional groups associated with the newly synthesized material. The characteristic peaks of pristine GO were observed at 3436, 1627, 1421, and $1068 \mathrm{~cm}^{-1}$ : each suggestive of stretching vibrations of hydroxyl functional groups associated with $\mathrm{GO}$ as well as free hydroxyl groups, $-\mathrm{C}=\mathrm{O}$ of the amide-I structure, carboxylate vibrations, and $-\mathrm{C}-\mathrm{O}$ stretching vibrations of carboxylic acids, respectively. The IR spectra analysis of GPM and the control reactions confirmed the hybrid reactions predicted in Fig. 1 and showed that EDC was effective in coupling EA to the GO sheets (Fig. S2, Supplementary Materials). Apart from the usual peaks of GO mentioned above, the intense peak at around $3400 \mathrm{~cm}^{-1}$ indicated the presence of additional free intermolecular -OH groups on the GPM material as well as the presence of $-\mathrm{NH}$ stretch. The intensity of this peak was however reduced on hydrazine reduction in rGPM due to the removal of some of 

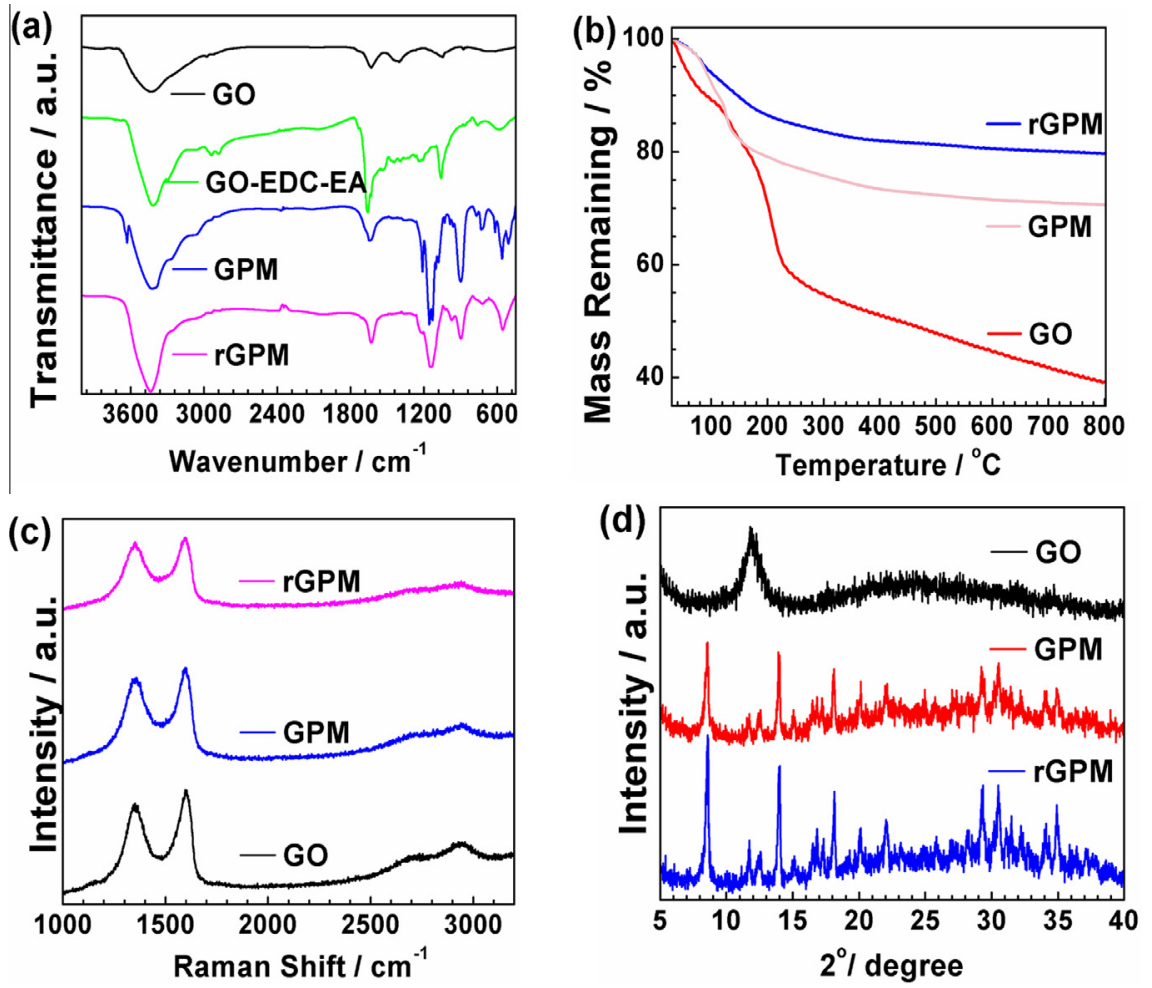

Fig. 2 - (a) IR spectra of GO, GO after reaction with EA (GO-EDC-EA), GPM, and rGPM. (b) TGA spectra of GO, GPM, and rGPM. (c) Raman spectra of GO, GPM, and rGPM. (d) XRD patterns of GO, GPM, and rGPM. (A color version of this figure can be viewed online.)

the $-\mathrm{OH}$ free groups. The appearance of the peak around $1210 \mathrm{~cm}^{-1}$ showed the presence of the $-\mathrm{C}-\mathrm{N}$ bond on the GPM materials: an indication of the successful reaction between the amine group of EA and carboxylic group of GO. The very strong peaks around $1640 \mathrm{~cm}^{-1}$ showed the presence of $-\mathrm{NH}$ bond of amide group on the new materials. The strong peaks of GPM and rGPM around 1152, 890, 730, and $560 \mathrm{~cm}^{-1}$ were all attributed to phosphate and phosphoramide groups.

TGA also confirmed the formation of the GPM. Fig. $2 b$ shows the weight loss for GO and GPM materials. Slight weight losses were observed for both samples at the first stage (at temperature $<105^{\circ} \mathrm{C}$ ). These losses were assigned to the residual water or absorbed solvent which were trapped within the assembly. The next stage of weight loss for GO was largely significant (35\%), occurred between 105 and $220^{\circ} \mathrm{C}$, and represents the pyrolysis and decomposition of the labile oxygen-containing functional groups such as $-\mathrm{CO},-\mathrm{OH}$ and $-\mathrm{COOH}$ attached to $\mathrm{GO}$ resulting in the loss of $\mathrm{CO}, \mathrm{CO}_{2}$ and steam. GPM on the other hand did not record such huge amount of weight loss (about 14\%) because these functional groups were involved in chemical reactions during the formation of the assembly. Further weight losses, especially for GPM, occurred gradually up till $400^{\circ} \mathrm{C}$ where the weight loss was nearly constant. rGPM showed similar weight loss pattern as GPM but with less final weight loss (9\%). Above $400^{\circ} \mathrm{C}$, the weight losses of GPM and rGPM were not significant and this indicated the material remaining at this temperature and beyond is stable. Thus, the GPM assembly was thermally stable.

The Raman spectra of pristine GO, GPM, and rGPM (Fig. 2c) showed that the GO structural skeleton remained intact after the functionalization. All three materials displayed two prominent peaks at around 1356 and $1596 \mathrm{~cm}^{-1}$, which corresponded to the well-documented $D$-and G-bands. The $D$ peaks were attributed to the defects in the graphene backbone structure, while the $G$ peaks were evidence of $s p^{2}$ bonded carbon that is present in planar sheet configurations which includes $s p^{2} \mathrm{C}=\mathrm{C}$ stretch vibrations in the GPM adsorbent. The D/G-intensity ratios of GO, GPM, and rGPM $(0.88$, 0.91 , and 0.93 , respectively), as well as their $D$ and $G$ bands linewidth were not significantly different from each other, an indication that the $\mathrm{C}=\mathrm{C}$ bonds were only slightly distorted and not involved in GPM formation. The variety of the D-band and $G$-band intensity ratio, reflecting the changes of the average size and number of the in-plane $s p^{2}$ domains, and the positional shifts were consistent with the reported results for chemically converted graphene $[14,15]$. The XRD pattern of the new GPM material confirmed the presence of TPP because it was consistent with that reported in literature [16]. The XRD peaks represent phosphate and phosphoramide groups that has been attached to the GO sheets.

The SEM image of the pristine GO (Fig. 3a) is significantly different from that of the GPM assembly (Fig. 3b). SEM image of GPM showed much rougher and smaller sheets than the pristine GO; and this was attributed to the presence of the 

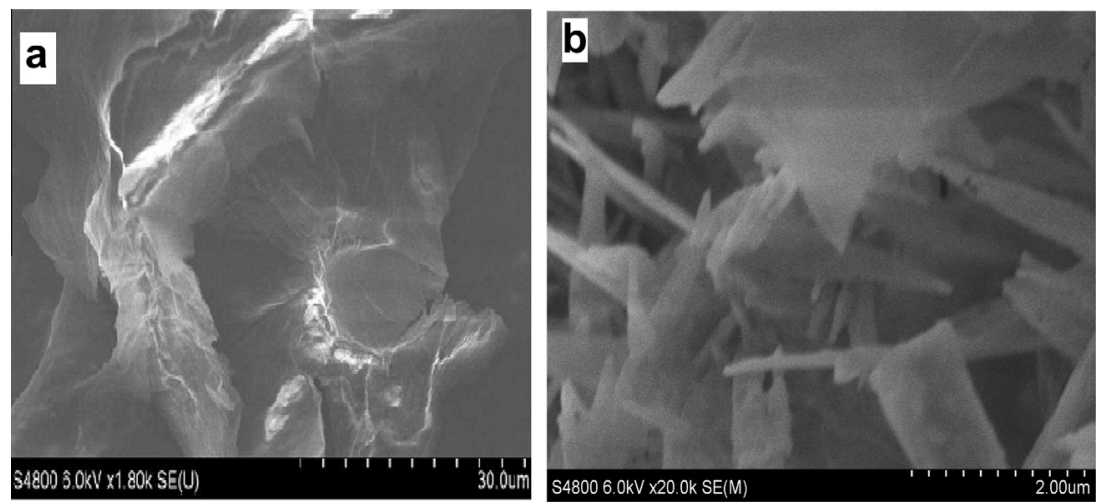

Fig. 3 - SEM images of (a) pristine GO showing unbroken sheets and (b) GPM showing rough and reduced sheets after the synthesis.

cross-linking groups such as TPP and EA on the surface of the GO sheets. The presence of these groups also contributed to the difference in the electrical density of the GPM assembly as opposed to graphene oxide sheets.

GO nanosheets dispersion usually aggregate upon drying due to stacking of the sheets. This led to the low BET surface area recorded which was significantly lower than theoretically predicted $2600 \mathrm{~m}^{2} \mathrm{~g}^{-1}$ [11]. Modification of GO sheets with rigid molecules usually reduce stacking of the sheets leading to higher values of BET surface area; however, we have used non-rigid and small molecules for the GO modification and cross-linking, and this has led to the reduced surface area of the new material. The BET surface area values of the freeze dried GO and GPM (Fig. S3, Supplementary Materials) was 56.2 and $41.5 \mathrm{~m}^{2} \mathrm{~g}^{-1}$, respectively. In addition, the pore diameter and pore volume of GPM also decreased from $4.9 \mathrm{~nm}$ and $0.06 \mathrm{~cm}^{3} \mathrm{~g}^{-1}$ to $4.6 \mathrm{~nm}$ and $0.04 \mathrm{~cm}^{3} \mathrm{~g}^{-1}$, respectively.

Preliminary investigation of dye adsorptions on GO and the various GO treatments (GPM and rGPM) showed that GPM assembly has adsorption capacity far higher than that of the pristine GO; Ramesha et al. [17] has also reported a similar low adsorption of cationic and anionic dyes by GO. In order to understand the details of the adsorption characteristics of GPM, further adsorption experiments were carried out.
The effect of time on the adsorption of any adsorbate in aqueous solution is an important parameter that helps in determining the efficiency of the adsorbent to be used for water treatment. Since industrial wastewater cannot be held in treatment plants for long periods, it is imperative that maximum sorption occurs as soon as possible. The optimum time for an adsorbent to be saturated by a sorbate can be determined by the time it takes $1.0 \mathrm{~g}$ of such adsorbent to reach maximum sorption. The adsorption time curve for BF obtained at pH 6.5 (Fig. 4a) shows that adsorption on GPM is very fast, reaching maximum in approximately $60 \mathrm{~min}$. This is consistent with the result obtained by Ramesha et al. [17] for dye adsorption by graphene based adsorbents. Thus, further experiments in this study have been incubated at $60 \mathrm{~min}$. This rapid adsorption trend is due to the high affinity of the cationic dyes on GPM because of electrostatic interaction between the positively charged amine group of $\mathrm{BF}$ and the negatively charged terminals of the phosphate groups as described in Figs. 1 and 6.

Fitting of the adsorption data to the two Lagergren kinetic models (PFO and PSO) showed that the adsorption data fit the PSO kinetics better than for PFO (Fig. S4, Supplementary Materials). This was evident in the correlation coefficient $\left(r^{2}\right)$ of the PSO (1.00) which was closer to unity than the PFO (0.64). This is an indication that the rate controlling
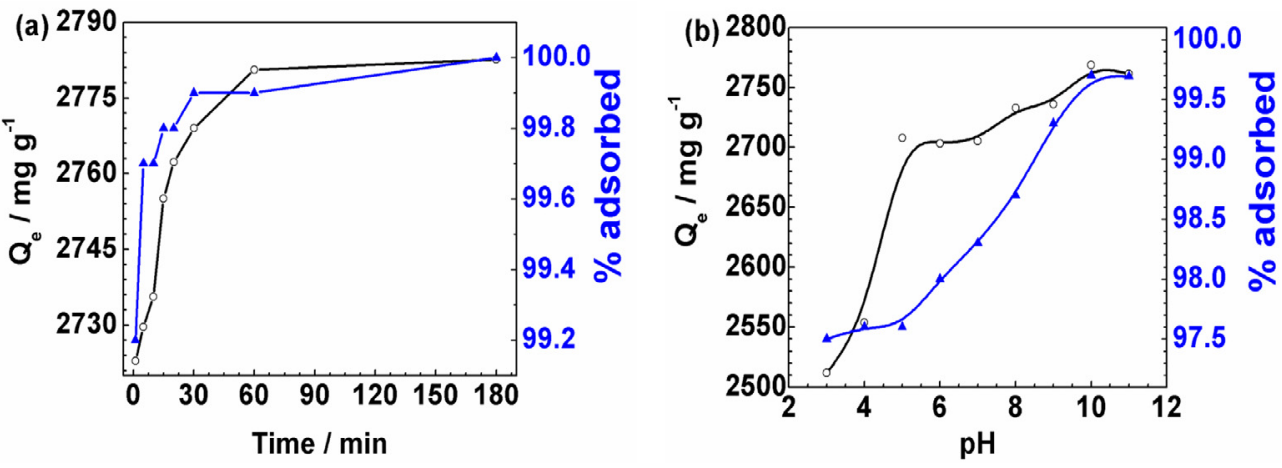

Fig. 4 - (a) Effect of time on BF adsorption by GPM. Black line denotes quantity of dye adsorbed ( $\left.Q_{e}\right)$ and the blue line shows the percentage (\%) adsorption. (b). Effect of $\mathrm{pH}$ on BF adsorption by GPM. Black line denotes quantity of dye adsorbed ( $\left.\mathrm{Q}_{e}\right)$ and the blue line shows the percentage (\%) adsorption. (A color version of this figure can be viewed online.) 
mechanism for adsorption involves valence forces through sharing or exchange of electrons between the GPM surfaces and the adsorbate - BF $[1,18]$.

Another important parameter used for establishing the adsorption behavior of a new adsorbent is the $\mathrm{pH}$ value of sorbate solution. This is imperative because the extent of adsorption of any adsorbate differs depending on the degree of charge density or electrostatic interaction surrounding either the adsorbate or adsorbent, or both, which in turn affects the degree of adsorption [1]. Hence, we have probed the effect of $\mathrm{pH}$ variation on $\mathrm{BF}$ adsorption in the $\mathrm{pH}$ range of 3 through 11 (Fig. 4b). The result showed that the adsorption from aqueous solution was $\mathrm{pH}$ dependent because as $\mathrm{pH}$ of the sorbate solution increased, the removal efficiency of GPM also increased until optimum $\mathrm{pH}$ for maximum adsorption which was observed in this study at $\mathrm{pH} 10$. This trend may be explained thus: $\mathrm{pH}$ affects the ionization of GPM functional groups (such as the phosphates) which are mainly responsible for the adsorptions. At very low $\mathrm{pH}$ values,
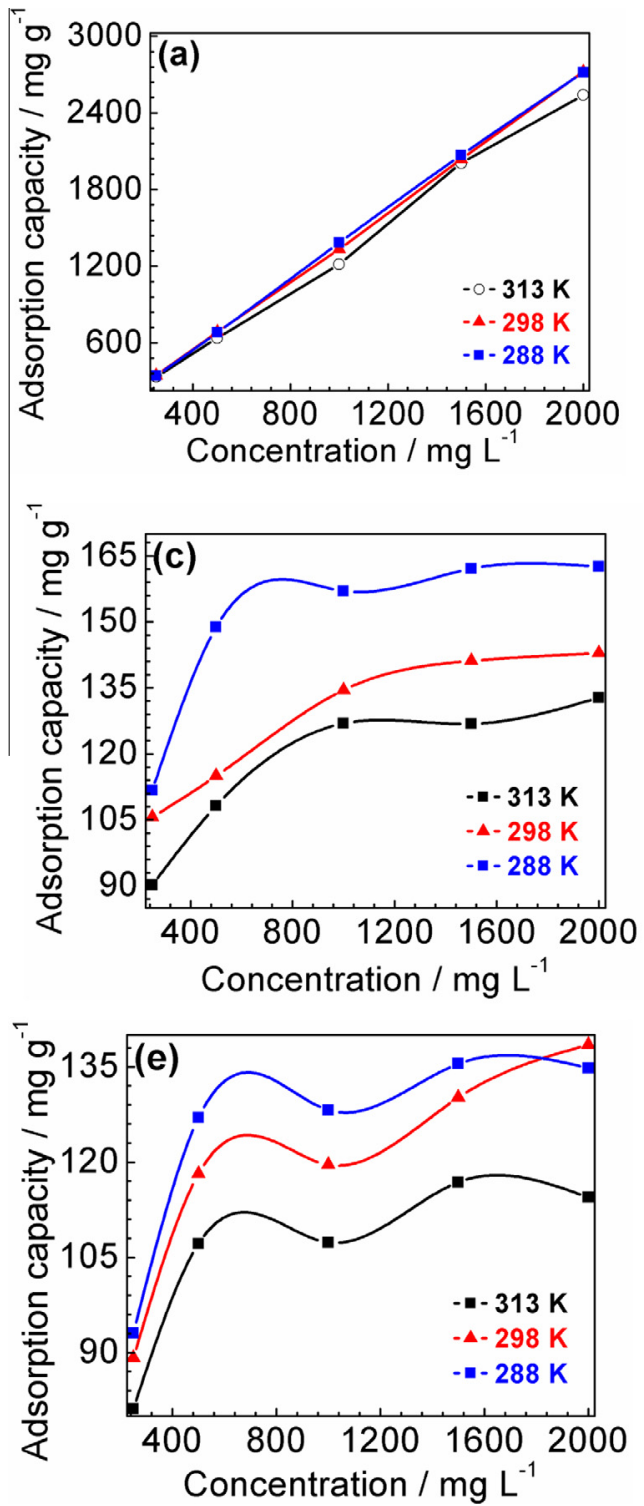

the extent of the ionization of the functional groups are very low, and the few groups that may ionize under this condition may not be available for adsorption because the stiff competition between the adsorbate and protons in solution. Hence, lower BF removal efficiencies were observed at lower $\mathrm{pH}$ values. However, as pH increased, functional groups ionization increased, and competitions from protons become less potent and are reduced drastically at above $\mathrm{pH}$ 7. This leads to increased negative charge density on the GPM which makes for better electrostatic interaction between negatively charged phosphate and the positively charged BF, leading to enhanced adsorptions; and hence the increased adsorptions observed which continued until optimum when virtually all adsorption sites were occupied at $\mathrm{pH}$ 10. Olu-Owolabi et al. [1] and Ramesha et al. [17] have reported similar results. Further experiments in this study were done at $\mathrm{pH} 10$.

The dye adsorption capacities of GPM for six dyes (MB, MO, $\mathrm{BF}, \mathrm{AM}, \mathrm{AY}$, and MV) were investigated at different initial concentrations $\left(250-2000 \mathrm{mg} \mathrm{L}^{-1}\right)$ and three temperatures $(288$,
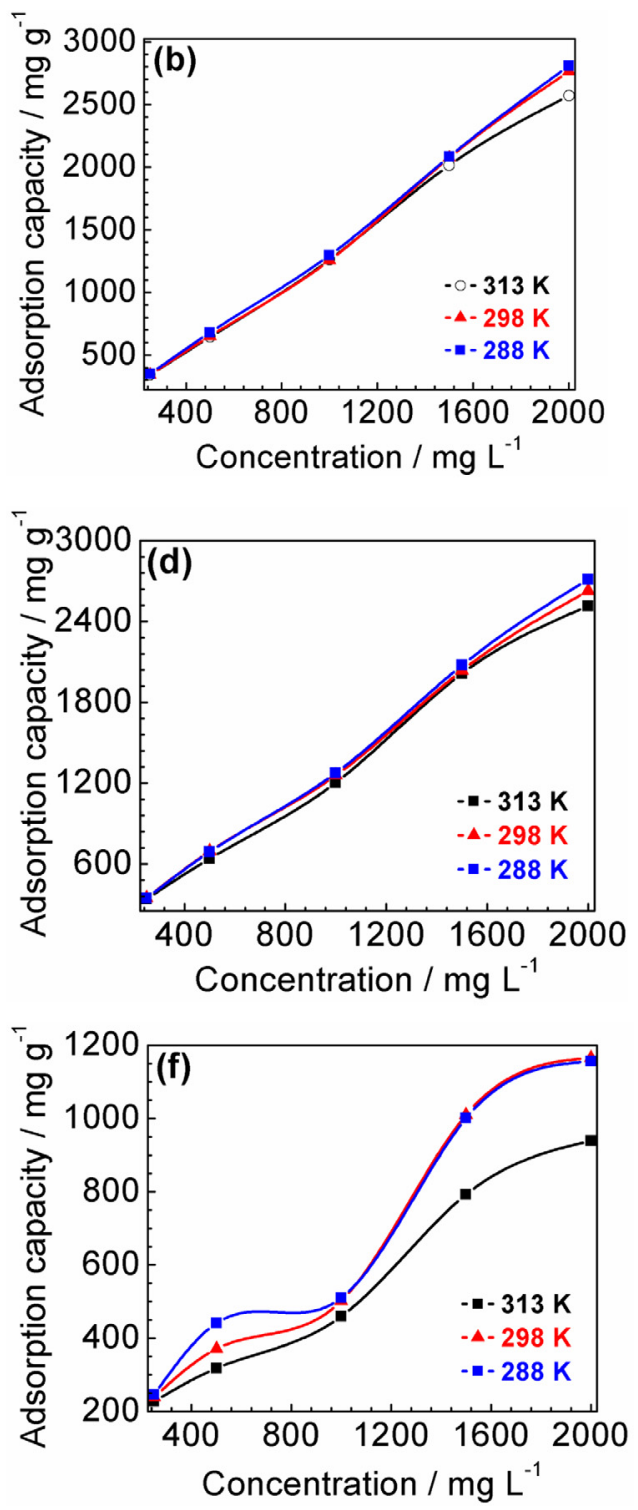

Fig. 5 - Adsorption trends of the various dyes at 288 (blue), 298 (red), and $313 \mathrm{~K}$ (black). (a) BF; (b) MB; (c) MO; (d) MV; (e) AY; and (f) AM. (A color version of this figure can be viewed online.) 
298, and $313 \mathrm{~K}$ ) (Fig. 5). The effect of temperature was not obvious on the adsorption curve (Fig. 5) up to concentrations of $1500 \mathrm{mg} \mathrm{L}^{-1}$ for adsorptions of the cationic dyes (BF, MB, and MV) since over $98 \%$ of the dyes in solution were removed; however, beyond this concentration, the effect became visible. Fig. S5 (Supplementary Materials) showed the color of dye solutions (BF, MB, and MV) before and after adsorptions. However, above this concentration and closer to GPM maximum adsorption capacity, lower temperatures favored the adsorptions; adsorption at $288 \mathrm{~K}$ was higher than at $313 \mathrm{~K}$. The adsorption trend at higher temperatures is $288>298>313 \mathrm{~K}$. The non-cationic dyes (MO, AY, and AM) showed varying adsorptions at different temperatures and different concentrations.
In terms of the adsorption capacity of GPM for these dyes, the trend was $\mathrm{BF} \geqslant \mathrm{MB} \geqslant \mathrm{MV} \gg \mathrm{AM}>\mathrm{MO}>\mathrm{AY}$. This trend was attributed basically to the charges on these dyes. The cationic dyes have high affinity to the negatively charged phosphate groups due to their positively charged amine group that brought about electrostatic interaction between these groups (Fig. 6). Molecular sieving could also be involved in these dyes' removal from solution because of the believed spongy nature of the GPM assembly. Hence, the mechanism of the cationic dyes' adsorptions involves electrostatic interactions, sieving, as well as $\pi-\pi$ interactions between the benzene rings in GPM and those of the dye (dye removal by sieving and $\pi-\pi$ interactions are limited processes). Similar observation has been reported by $\mathrm{Xu}$ et al. [19] and

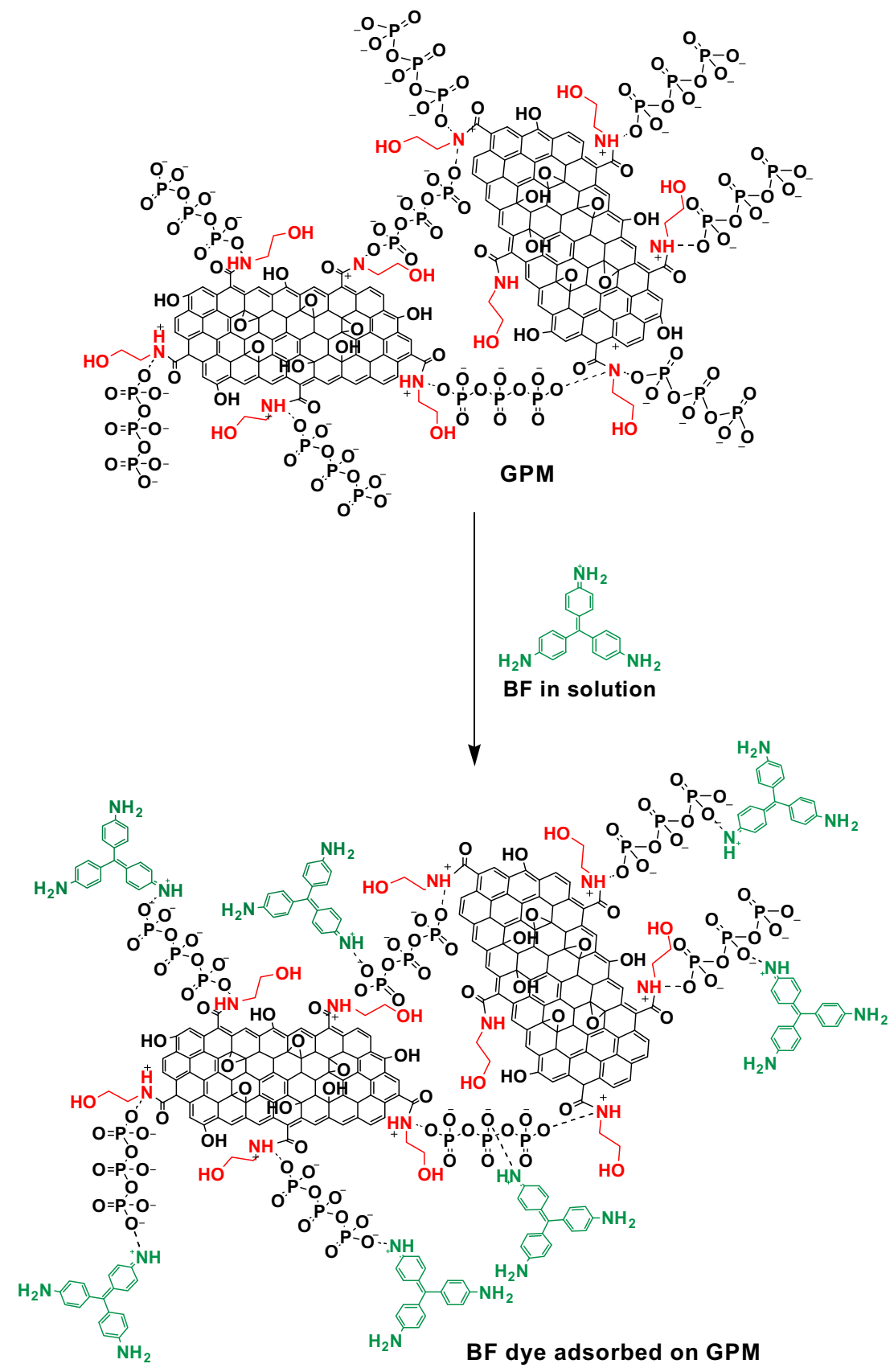

Fig. 6 - Schematics for the main adsorption mechanism for cationic dyes using BF as an example. (A color version of this figure can be viewed online.) 
Table 1 - BF adsorption thermodynamic parameters at 288, 298, and $313 \mathrm{~K}$.

\begin{tabular}{llll} 
Temperature $(\mathrm{K})$ & 288 & 298 & \multicolumn{1}{l}{313} \\
\hline$\Delta G^{\circ}\left(\mathrm{kJ} \mathrm{mol}^{-1}\right)$ & -14.78 & -14.97 & -14.34 \\
$\Delta H^{\circ}\left(\mathrm{kJ} \mathrm{mol}^{-1}\right)$ & -0.34 & -20.34 & -20.34 \\
$\Delta S^{\circ}\left(\mathrm{kJ} \mathrm{K}^{-1} \mathrm{~mol}^{-1}\right)$ & -0.02 & -0.02 & -0.02 \\
\hline
\end{tabular}

\begin{tabular}{|c|c|c|c|}
\hline Parameter & $288 \mathrm{~K}$ & $298 \mathrm{~K}$ & $313 \mathrm{~K}$ \\
\hline \multicolumn{4}{|l|}{ Langmuir } \\
\hline${ }^{*} Q_{0}$ & 1012.11 & 989.12 & 1000.00 \\
\hline$r^{2}$ & 0.261 & 0.653 & 0.553 \\
\hline \multicolumn{4}{|l|}{ Freundlich } \\
\hline$K_{f}$ & 571.48 & 493.17 & 295.80 \\
\hline$r^{2}$ & 0.942 & 0.960 & 0.704 \\
\hline
\end{tabular}

Olu-Owolabi et al. [20]. The non-cationic dyes on the other hand cannot be removed by electrostatic interaction because they lack a corresponding positively charged group in their structure, and thus sieving and $\pi-\pi$ interactions are main driving forces responsible of anionic dyes' removal from solution.

It is assumed that in an isolated system, energy cannot be gained or lost and the entropy change is the only driving force. However, in environmental application, both energy and entropy must be considered in order to determine which process(es) will occur spontaneously. Hence, using BF as a representative cationic dye, the adsorption thermodynamic parameters were calculated (Supplementary Materials) and shown in Table 1. The adsorption on GPM was spontaneous (negative $\Delta G^{\circ}$ ) and exothermic (negative $\Delta H^{\circ}$ ), with decrease in the randomness at the adsorbent-solution interface during the reaction (negative $\Delta S^{\circ}$ ). This is a reflection of the dye affinity to GPM surface at equilibrium. The exothermic nature of the adsorption was buttressed by the reduction in the adsorption as the temperature increased.

Fitting of the adsorption data to the Langmuir and Freundlich adsorption isotherms (Table 2) showed that the adsorption was better described by the Freundlich isotherm at all temperatures, with $r^{2}$ values that were closer to unity than the Langmuir isotherm. The Langmuir isotherm assumes that the sorption sites on GPM possess equal affinity for the dye, and forms a monolayer on GPM surface at saturation; but the Freundlich isotherm assumes sorption on heterogeneous surfaces [1]. The fit of the adsorption data to the Freundlich isotherm implies that in addition to $\pi-\pi$ interactions (between the aromatic rings of GPM and those of the dyes), electrostatic interactions (between the charged surfaces of GPM and the dyes), and molecular sieving (of the dyes by the GPM), multi-layer adsorptions (resulting from $\pi-\pi$ interactions between the aromatic rings of the surface adsorbed dyes and dyes in solution) are also involved in these dyes removals from solution. The poor fitting of the Freundlich adsorption isotherm obtained at $313 \mathrm{~K}$ was attributed to inherent errors associated with using the linear model calculations of the Freundlich adsorption isotherm parameters which become significant when dealing with low energy adsorption surfaces like the GPM.

Comparison of the GPM adsorption capacity with some of those reported in literature (Table 3) showed that GPM is a very potent adsorbent for cationic dyes.

\section{Conclusions}

This study illustrated the simple synthesis of graphene oxide tripolyphosphate material under benign conditions. The tripolyphosphate group conferred a very environmentally useful property to the graphene - superchelating capacity. IR, TGA, Raman shift, SEM, XRD, and surface area confirmed the formation of the assembly. Graphene oxide tripolyphosphate

\section{Table 3 - Review of maximum sorption capacities $\left(Q_{0}\right)$ of some sorbents for some similar dyes.}

\begin{tabular}{|c|c|c|c|}
\hline Adsorbent & Adsorbate & $Q_{0}\left(\mathrm{mg} \mathrm{g}^{-1}\right)$ & Refs. \\
\hline Thermal power plant fly ash & Crystal violet & 4.6 & {$[21]$} \\
\hline Bottom ash (power plant waste) & $\mathrm{BF}$ & 7.2 & {$[22]$} \\
\hline Jalshakti & MB & 11.7 & [23] \\
\hline De-oiled soya & $\mathrm{BF}$ & 13.5 & [22] \\
\hline$N$-(2-aminoethyl) amino dexoxy $\beta$-cyclodextrin polymer & $\mathrm{BF}$ & 16.5 & [24] \\
\hline$\beta$-cyclodextrin polymer ( $\beta$-CDP) & $\mathrm{BF}$ & 32.3 & [24] \\
\hline Hydroxyl-propyl $\beta$-cyclodextrin polymer (HP- $\beta$-CDP) & $\mathrm{BF}$ & 39.0 & {$[24]$} \\
\hline Carboxyl-methyl $\beta$-cyclodextrin polymer (CM- $\beta$-CDP) & $\mathrm{BF}$ & 54.6 & [24] \\
\hline Jute fiber carbon & Malachite green & 136.6 & {$[25]$} \\
\hline GPM & AY & 138.5 & Present study \\
\hline GPM & MO & 142.9 & Present study \\
\hline Phosphoric acid modified rice straw & Basic blue 9 & 208.33 & [26] \\
\hline Fe nanoparticles/graphene composite (F1G1) & $\mathrm{MB}$ & 250.0 & [27] \\
\hline Fe nanoparticles/graphene composite (F5G1) & MB & 416.7 & [27] \\
\hline Graphene oxide & & 673.0 & Present study \\
\hline GPM & AM & 1165.6 & Present study \\
\hline GPM & MV & 2627.5 & Present study \\
\hline GPM & $\mathrm{BF}$ & 2722.7 & Present study \\
\hline GPM & MB & 2761.5 & Present study \\
\hline
\end{tabular}


material shows a potent and fast cationic dye removal that uses electrostatic, sieving, and $\pi-\pi$ interactions to remove dyes from solution. The removal is spontaneous and exothermic, and can be described by the pseudo-second order kinetics as well as Freundlich isotherm. The experimental maximum adsorption capacity of the material for cationic dyes (>2540 $\mathrm{mg} \mathrm{g}^{-1}$ ) far exceeds those reported in literature. Hence, for GPM to be useful for cleanup of wastewater containing reactive dye, the reactive dye must have at least a cationic moiety.

\section{Acknowledgements}

The financial support of the National Science Foundation of China (Grants no. 21374024 and 61261130092) and the Ministry of Science and Technology of China (Grant 2014CB932200) is acknowledged. We also acknowledge the supports of Chief S.L. Edu/Chevron Research grant, Nigeria and the World Academy of Sciences (TWAS), Trieste Italy and the Chinese Academy of Sciences (CAS) for the award of CAS-TWAS Postgraduate Fellowship (FR number: 3240255024) to P.N.D.

\section{Appendix A. Supplementary data}

Supplementary data associated with this article can be found, in the online version, at http://dx.doi.org/10.1016/ j.carbon.2014.07.057.

\section{R E F E R E N C E S}

[1] Olu-Owolabi BI, Diagboya PN, Ebaddan WC. Mechanism of $\mathrm{Pb}^{2+}$ removal from aqueous solution using a nonliving moss biomass. Chem Eng J 2012;195-196:270-5.

[2] Allen MJ, Tung VC, Kaner RB. Honeycomb carbon: a review of graphene. Chem Rev 2010;110(1):132-45.

[3] Lemme MC, Echtermeyer TJ, Baus M, Kurz HA. Graphene field effect device. IEEE Electron Device Lett 2007;28(4):1-12.

[4] Schedin F, Geim AK, Morozov SV, Hill EW, Blake P, Katsnelson MI, et al. Detection of individual gas molecules adsorbed on graphene. Nat Mater 2007;6(9):652-5.

[5] Yang Y, Wang J, Zhang J, Liu J, Yang X, Zhao H. Exfoliated graphite oxide decorated by PDMAEMA chains and polymer particles. Langmuir 2009;25(19):11808-14.

[6] Liu Z, Robinson JT, Sun X, Dai H. PEGylated nanographene oxide for delivery of water-insoluble cancer drugs. J Am Chem Soc 2008;130(33):10876-7.

[7] Lee B, Chen Y, Duerr F, Mastrogiovanni D, Garfunkel E, Andrei EY, et al. Modification of electronic properties of graphene with self-assembled monolayers. Nano Lett 2010;10(7):2427-32.

[8] Hummers WS, Offeman RE. Preparation of graphitic oxide. J Am Chem Soc 1958;80(6):1339.
[9] Hirata M, Gotou T, Horiuchi S, Fujiwara M, Ohba M. Thin-film particles of graphite oxide 1: high-yield synthesis and flexibility of the particles. Carbon 2004;42(14):2929-37.

[10] Cheng QY, Zhou D, Gao Y, Chen Q, Zhang Z, Han BH. Supramolecular self-assembly induced graphene oxide based hydrogels and organogels. Langmuir 2012;28(5):3005-10.

[11] Dreyer DR, Park S, Bielawski CW, Ruoff RS. The chemistry of graphene oxide. Chem Soc Rev 2010;39(1):228-40.

[12] Salavagione HJ, Martinez G, Ellis G. Recent advances in the covalent modification of graphene with polymers. Macromol Rapid Commun 2011;32(22):1771-89.

[13] Lee ST, Mi FL, Shen YJ, Shyu SS. Equilibrium and kinetic studies of copper(II) ion uptake by chitosan-tripolyphosphate chelating resin. Polymer 2001;42:1879-92.

[14] Zhou D, Han BH. Graphene-based nanoporous materials assembled by mediation of polyoxometalate nanoparticles. Adv Funct Mater 2010;20(16):2717-22.

[15] Wu CQ, Cheng SS, Han BH. Templated patterning of graphene oxide using self-assembled monolayers. Carbon 2012;50(3):1083-9.

[16] Mabis AJ, Quimby OT. Analysis of sodium pyro- and tripolyphosphate mixtures by X-Ray diffractometer using an internal standard. Anal Chem 1953;25:1814-8.

[17] Ramesha GK, Kumara AV, Muralidhara HB, Sampath S. Graphene and graphene oxide as effective adsorbents toward anionic and cationic dyes. J Coll Interf Sci 2011;361(1):270-7.

[18] Feng N, Guo X, Liang S, Zhu Y, Liu J. Biosorption of heavy metals from aqueous solutions by chemically modified orange peel. J Hazard Mater 2011;185(1):49-54.

[19] Xu J, Wang L, Zhu Y. Decontamination of bisphenol a from aqueous solution by graphene adsorption. Langmuir 2012;28(22):8418-25.

[20] Olu-Owolabi BI, Diagboya PN, Adebowale KO. Evaluation of pyrene sorption-desorption on tropical soils. J Environ Manage 2014;137:1-9.

[21] Mohan D, Singh KP, Singh G, Kumar K. Removal of dyes from wastewater using fly ash, a low-cost adsorbent. Ind Eng Chem Res 2002;41(15):3688-95.

[22] Gupta VK, Mittal A, Gajbe V, Mittal J. Adsorption of basic fuchsin using waste materials - bottom ash and deoiled soya - as adsorbents. J Coll Interf Sci 2008;319(1):30-9.

[23] Dhodapkar R, Rao NN, Pande SP, Nandy T, Devotta S. Adsorption of cationic dyes on Jalshakti ${ }^{\oplus}$, super absorbent polymer and photocatalytic regeneration of the adsorbent. React Funct Polym 2007;67(6):540-8.

[24] Zhang XM, Peng CS, Xu GC. Synthesis of modified $\beta$ cyclodextrin polymers and characterization of their fuchsin adsorption. J Incl Phenom Macrocycl Chem 2011;72(1-2): 165-71.

[25] Porkodi K, Vasanth KK. Equilibrium, kinetics and mechanism modeling and simulation of basic and acid dyes sorption onto jute fiber carbon: eosin yellow, malachite green and crystal violet single component systems. J Hazard Mat 2007;143(1-2):311-27.

[26] Gong R, Jin Y, Chen J, Hu Y, Sun J. Removal of basic dyes from aqueous solution by sorption on phosphoric acid modified rice straw. Dyes Pigments 2007;73(3):332-7.

[27] Guo J, Wang R, Tjiu WW, Pan J, Liu T, Synthesis of Fe nanoparticles@graphene composites for environmental applications. J Hazard Mat 2012; 225-226: 63-73. 